PROCEEDINGS OF THE

AMERICAN MATHEMATICAL SOCIETY

Volume 131, Number 1, Pages 201-207

S 0002-9939(02)06530-9

Article electronically published on May 22, 2002

\title{
WEIGHTED HOLOMORPHIC SPACES WITH TRIVIAL CLOSED RANGE MULTIPLICATION OPERATORS
}

\author{
KINGA CICHOŃ AND KRISTIAN SEIP
}

(Communicated by Joseph A. Ball)

\begin{abstract}
We deal with the space $H_{v}^{\infty}$ consisting of those analytic functions $f$ on the unit disc $\mathbb{D}$ such that $\|f\|_{v}:=\sup _{z \in \mathbb{D}} v(z)|f(z)|<\infty$, with $v(z)=$ $v(|z|)$. We determine the critical rate of decay of $v$ such that the pointwise multiplication operator $M_{\varphi}, M_{\varphi}(f)(z)=\varphi(z) f(z)$ and $\varphi$ analytic, has closed range in $H_{v}^{\infty}$ only in the trivial case that $\varphi$ is the product of an invertible function in $H^{\infty}$ and a finite Blaschke product.
\end{abstract}

In this note, we deal with pointwise multiplication operators $M_{\varphi}, M_{\varphi}(f)(z):=$ $\varphi(z) f(z)$, where $\varphi: \mathbb{D} \rightarrow \mathbb{C}$ denotes a bounded non-constant analytic function on the unit disc $\mathbb{D}$. We let $M_{\varphi}$ act on weighted Banach spaces of the following form:

$$
H_{v}^{\infty}:=H_{v}^{\infty}(\mathbb{D}):=\left\{f \in H(\mathbb{D}):\|f\|_{v}:=\sup _{z \in \mathbb{D}} v(z)|f(z)|<\infty\right\} .
$$

Here $H(\mathbb{D})$ denotes the space of analytic functions on $\mathbb{D}$ and $v: \mathbb{D} \rightarrow \mathbb{R}^{+}$is an arbitrary weight, i.e., a continuous strictly positive function such that $H_{v}^{\infty}$ contains a non-zero function. We will consider only radial weights $v$, i.e., we assume $v(z)=$ $v(|z|)$.

We are interested in knowing when $M_{\varphi}$ has closed range in $H_{v}^{\infty}$, i.e., when there exists a positive constant $C$ such that $\left\|M_{\varphi} f\right\|_{v} \geq C\|f\|_{v}$ for all $f \in H_{v}^{\infty}$. It is clear that no matter what $v$ is, if $\varphi=h b$ with $h$ an invertible function in $H^{\infty}$ and $b$ a finite Blaschke product, then trivially $M_{\varphi}$ has closed range. The purpose of this note is to determine when this trivial case is the only one allowed by $v$. As one might expect, this amounts to finding a certain critical rate of decay of $v$.

A few words on the background of this problem are in order. Pointwise multiplication operators between different Bergman spaces have been studied by many authors; see for example, [A], L1], L2, $\mathrm{MS}$, and [V]. In particular, McDonald and Sundberg [MS] were interested in the exact behavior of the multiplication operator when $\varphi$ is an inner function. Luecking [L1] determined when the pointwise multiplication operators $M_{\varphi}$ have closed range when acting on the weighted Bergman spaces $A_{v}^{p}(\mathbb{D}):=\left\{f \in H(\mathbb{D}): \quad\|f\|_{v}^{p}:=\int_{\mathbb{D}}|f(z)|^{p} v(z) d A(z)<\infty\right\}$, if $1 \leq p<\infty$, where $d A(z)$ is the Lebesgue area measure and $v(z):=\left(1-|z|^{2}\right)^{\alpha}$, $0<\alpha<\infty$, or $v(z) \equiv 1$. Luecking's result was extended to the weighted Bergman spaces $A_{v}^{\infty}(\mathbb{D}):=H_{v}^{\infty}(\mathbb{D})$ by Bonet, Domański and Lindström in [BDL2], with more

Received by the editors April 17, 2001 and, in revised form, August 21, 2001.

2000 Mathematics Subject Classification. Primary 47B38.

Key words and phrases. Weighted Banach space of analytic functions, pointwise multiplication operator, closed range. 
general weights $v$ satisfying

$$
\frac{A}{\left(1-|z|^{2}\right)^{2}} \leq-\Delta \log v(z) \leq \frac{B}{\left(1-|z|^{2}\right)^{2}} ;
$$

here $\Delta$ denotes the Laplacian and $A, B$ are positive constants. This condition on the Laplacian appears in $[\mathrm{BO}$, and $[\underline{\mathrm{S}}$. By an approximation theorem in the latter paper, the condition ensures that the spaces $H_{v}^{\infty}$ have function theoretic properties that essentially coincide with those of the Bergman spaces studied by Luecking. For such weights, $M_{\varphi}$ has closed range if and only if $\varphi=h b$, with $h$ an invertible function in $H^{\infty}$ and $b$ a finite union of interpolating Blaschke products BDL2. It was conjectured in BDL2] that if $-\left(1-|z|^{2}\right)^{2} \Delta \log v(z) \rightarrow+\infty$ when $|z| \rightarrow 1^{-}$, then $M_{\varphi}$ has closed range only in the trivial case $\varphi=h b$ with $h$ an invertible function in $H^{\infty}$ and $b$ a finite Blaschke product. The present note confirms this conjecture:

Theorem 1. Let $v \in C^{2}(\mathbb{D})$ be a radial weight such that

$$
-\left(1-|z|^{2}\right)^{2} \Delta \log v(z) \rightarrow+\infty \quad \text { as } \quad|z| \rightarrow 1^{-} .
$$

Then $M_{\varphi}: H_{v}^{\infty} \rightarrow H_{v}^{\infty}$ has closed range if and only if $\varphi=h b$, where $h$ is invertible in $H^{\infty}$ and $b$ is a finite Blaschke product.

The weights covered by Theorem 1 are typically weights tending to 0 faster than any of the weights $v(z)=\left(1-|z|^{2}\right)^{\alpha}, \alpha>0$, when $|z| \rightarrow 1^{-}$. The conclusion of Theorem 1 has previously been obtained under the stronger assumption that $v$ tends exponentially to zero at the boundary $\left[\mathrm{B}\right.$. (A typical case is $v(z)=\exp \left(-\frac{1}{\left(1-|z|^{2}\right)^{\delta}}\right)$, $\delta>0$.)

It was shown in BDL2] that the problem of determining those $\varphi$ such that $M_{\varphi}$ has closed range in $H_{v}^{\infty}$ has a nice link to the theory of uniform algebras. Let $M\left(H^{\infty}\right)$ be the maximal ideal space of $H^{\infty}$, and let $\Gamma\left(H^{\infty}\right)$ denote the Shilov boundary of $H^{\infty}$. It was proved in BDL2 that for any fixed weight $v$ there is a closed subset $A_{v}$ of $M\left(H^{\infty}\right), \Gamma\left(H^{\infty}\right) \subset A_{v} \subset M\left(H^{\infty}\right) \backslash \mathbb{D}$, such that $M_{\varphi}$ has closed range in $H_{v}^{\infty}$ if and only if $\varphi$ does not vanish on $A_{v}$. Theorem 1 identifies the extreme case $A_{v}=M\left(H^{\infty}\right) \backslash \mathbb{D}$, while [BDL2] deals with the case that $\varphi$ does not vanish on any trivial Gleason part. An interesting question is whether there exists a weight $v$ for any closed set $A, \Gamma\left(H^{\infty}\right) \subset A \subset M\left(H^{\infty}\right) \backslash \mathbb{D}$, such that $A_{v}=A$.

The remainder of this note is devoted to the proof of Theorem 1 . We begin with some explicit calculations.

Proposition 2. Let $f: \mathbb{D} \rightarrow \mathbb{R}_{+}$be a continuous radial function. Then the only radial solution $\psi \in C^{2}(\mathbb{D})$ of the equation

$$
\Delta \psi(z)=f(z)
$$

satisfying $\psi(0)=0$ is the following:

$$
\psi(z)=\int_{0}^{|z|} r f(r)(\log |z|-\log r) d r .
$$

Proof. We recall that the Laplacian has the following representation in polar coordinates $(t, \theta)$ :

$$
\Delta \psi(t, \theta)=\frac{\partial^{2} \psi}{\partial t^{2}}+\frac{1}{t} \frac{\partial \psi}{\partial t}+\frac{1}{t^{2}} \frac{\partial^{2} \psi}{\partial \theta^{2}}
$$


Since $\psi$ is a radial function, we have to find the solution of the non-homogenous Cauchy-Euler differential equation:

$$
\left(\frac{d^{2}}{d t^{2}}+\frac{1}{t} \frac{d}{d t}\right) \psi(t)=f(t)
$$

It is plain that for $z \neq 0$

$$
\psi(z)=\int_{0}^{|z|} r f(r)(\log |z|-\log r) d r
$$

satisfies the above equation. Hence,

$$
\lim _{|z| \rightarrow 0} \psi(z)=0 .
$$

A calculation shows that $\Delta \psi(0)=f(0)$. Thus a general solution of the equation is

$$
\psi_{g}(z)=\psi(z)+u(z),
$$

where $u$ is a harmonic function. But the mean value property of harmonic functions shows that the only radial harmonic functions are constant functions, and so $u(z) \equiv$ 0 .

Given a continuous function $f$ on $[0,1)$ and some $\eta \in(0,1]$, we define $p(t):=$ $\frac{1-\eta t}{t} \int_{0}^{t} r f(r) d r$ for $0 \leq t<1$.

Lemma 3. Let $f:[0,1) \rightarrow \mathbb{R}_{+}$be a continuous function. If $\left(1-t^{2}\right)^{2} f(t) \nearrow+\infty$ as $t \rightarrow 1^{-}$, then $p(t) \nearrow+\infty$ as $t \rightarrow 1^{-}$.

Proof. Since

$$
\begin{aligned}
p(t) & \geq(1-t) \int_{2 t-1}^{t} r f(r) d r \\
& \geq \frac{1}{4}(1-t)(3 t-1)\left(1-(2 t-1)^{2}\right) f(2 t-1) \text { for } t \text { near } 1,
\end{aligned}
$$

it is immediate that $p(t) \rightarrow \infty$ as $t \rightarrow 1^{-}$. So we need only check that $p^{\prime}(t) \geq 0$ for all $t$. We compute

$$
p^{\prime}(t)=-\frac{1}{t^{2}} \int_{0}^{t} r f(r) d r+(1-\eta t) f(t) .
$$

By the assumption on $f$, we have

$$
f(r) \leq f(t) \frac{\left(1-t^{2}\right)^{2}}{\left(1-r^{2}\right)^{2}}
$$

for each $r \leq t$. Hence,

$$
\frac{1}{t^{2}} \int_{0}^{t} r f(r) d r \leq \frac{\left(1-t^{2}\right)^{2} f(t)}{t^{2}} \int_{0}^{t} \frac{r d r}{\left(1-r^{2}\right)^{2}} \leq(1-t) f(t) \leq(1-\eta t) f(t) .
$$

It follows that

$$
p^{\prime}(t) \geq(1-\eta t) f(t)-(1-t) f(t)=t(1-\eta) f(t) .
$$

In particular, $p^{\prime}(t) \geq 0$. 
We define the lower norm of the operator $T: X \rightarrow Y, X, Y$ Banach spaces as

$$
L(T):=\inf \{\|T f\|:\|f\|=1\} .
$$

The operator $T$ has closed range if and only if $L(T)>0$. We set $\varphi_{\eta}(z):=\frac{\eta-z}{1-\bar{\eta} z}$ for $\eta, z \in \mathbb{D}$ and $\rho(z, \eta)=\left|\varphi_{\eta}(z)\right|$. We now establish the main ingredient in the proof of Theorem 1.

Lemma 4. Let $w \in C^{2}(\mathbb{D})$ be a radial weight and set $-\Delta \log w(z)=f(z)$. If

$$
\left(1-t^{2}\right)^{2} f(t) \nearrow+\infty \quad \text { as } t \rightarrow 1^{-} \text {, }
$$

then the lower norm of $M_{\varphi_{\eta}}: H_{w}^{\infty} \rightarrow H_{w}^{\infty}$ tends to 0 as $|\eta| \rightarrow 1^{-}$.

Proof. We make some preliminary estimates. By Proposition 2, we have that

$$
w(z)=\exp \left\{\int_{0}^{|z|} r f(r)(\log r-\log |z|) d r\right\} .
$$

Since the weight $w$ is radial, all rotations are isometries, so without loss of generality we may assume that $\eta$ is a positive real number.

We fix an $\eta \in(0,1)$, and define $F_{\eta}(z):=\frac{a(\eta)}{(1-\eta z)^{\alpha(\eta)}}$, where $\alpha(\eta)>1$ and $a(\eta)$ are chosen in such a way that the function

$$
H(z)=\log \left|F_{\eta}(z) w(z)\right|
$$

satisfies $H(\eta)=H^{\prime}(\eta)=0$. Since

$$
H^{\prime}(t)=\frac{\alpha(\eta) \eta}{1-\eta t}-\frac{1}{t} \int_{0}^{t} r f(r) d r
$$

we obtain

$$
\alpha(\eta)=\frac{1-\eta^{2}}{\eta^{2}} \int_{0}^{\eta} r f(r) d r
$$

Thus

$$
H^{\prime}(t)=\frac{1}{(1-\eta t)}\left[\frac{1-\eta^{2}}{\eta} \int_{0}^{\eta} r f(r) d r-\frac{1-\eta t}{t} \int_{0}^{t} r f(r) d r\right]
$$

which may be written

$$
H^{\prime}(t)=\frac{1}{(1-\eta t)}[p(\eta)-p(t)]
$$

From Lemma 3 , it is seen that $H^{\prime}(t)>0$ for $t<\eta$ and $H^{\prime}(t)<0$ for $t>\eta$. Hence the function $H(t)$ has only one maximum $\eta$, increases for $t<\eta$ and decreases for $t>\eta$. Thus $\left\|F_{\eta}\right\|_{w}=1$.

By (2), we may write

$$
H^{\prime}(t)=\frac{1}{(1-\eta t)} \int_{t}^{\eta} p^{\prime}(x) d x .
$$

It then follows from inequality (1) that

$$
\left|H^{\prime}(t)\right|=\frac{1}{(1-\eta t)}\left|\int_{t}^{\eta} p^{\prime}(x) d x\right| \geq \frac{(1-\eta)}{(1-\eta t)}\left|\int_{t}^{\eta} x f(x) d x\right| .
$$

We set $\tau=\min (t, \eta)$, so that we obtain

$$
\left|H^{\prime}(t)\right| \geq \frac{(1-\eta)}{(1-\eta t)} f(\tau)\left|\int_{t}^{\eta} x d x\right|=\frac{(1-\eta)}{(1-\eta t)} f(\tau) \frac{\left|t^{2}-\eta^{2}\right|}{2} .
$$


We now use (3) and the assumption on $f$ to prove that there exists a function $\epsilon(\eta)>0$ such that $\epsilon(\eta) \rightarrow 0$ and $\sup _{\rho(z, \eta) \geq \epsilon(\eta)} H(z) \rightarrow-\infty$ when $\eta \rightarrow 1^{-}$. We will prove this statement in the following form. Suppose $\delta(\eta)$ is such that $\delta(\eta) /(1-\eta) \rightarrow$ 0 , and define

$$
Q_{\eta, \delta}=\left\{z=t e^{i \theta}:|t-\eta|<\delta(\eta),|\theta| \leq \delta(\eta)\right\} .
$$

We claim that $\sup _{z \notin Q_{\eta, \delta}} H(z) \rightarrow-\infty$ when $\eta \rightarrow 1^{-}$for a suitable function $\delta$, depending on $f$. It is clear that this statement proves the lemma, $\operatorname{since} \sup _{z \in Q_{\eta, \delta}} \varphi_{\eta}(z)$ $\rightarrow 0$ when $\eta \rightarrow 1^{-}$.

To prove the claim, we now pick $\delta$. We may assume that $\eta>1 / 2$. By monotonicity of $t \mapsto H(t)$ for (respectively) $t<\eta$ and $t>\eta$, and of $\theta \mapsto H\left(t e^{i \theta}\right)$ for (respectively) $\theta>0$ and $\theta<0$, the supremum of $H$ outside $Q_{\eta, \delta}$ will be attained on the boundary of $Q_{\eta, \delta}$. In particular, we may thus assume that $|t-\eta|<(1-\eta) / 2$. Then (3) gives

$$
H(t)=\int_{\eta}^{t} H^{\prime}(\xi) d \xi \leq-C(\eta) \frac{(t-\eta)^{2}}{(1-\eta)^{2}}
$$

with

$$
C(\eta)=\frac{1}{20}(1-\eta)^{2} f(\eta-(1-\eta) / 2)
$$

By the growth assumption on $f, C(\eta) \rightarrow+\infty$ when $\eta \rightarrow 1^{-}$. Thus we may require

$$
C(\eta) \frac{[\delta(\eta)]^{2}}{(1-\eta)^{2}} \rightarrow \infty
$$

In addition, we will require that for $|t-\eta| \leq \delta(\eta)$ and $|\theta| \geq \delta(\eta)$, we have

$$
\log \left|F_{\eta}(t)\right|-\log \left|F_{\eta}\left(t e^{i \theta}\right)\right|=-\alpha(\eta) \log \left|\frac{1-\eta t}{1-\eta t e^{i \theta}}\right| \rightarrow \infty
$$

when $\eta \rightarrow 1^{-}$. Since $\alpha(\eta) \rightarrow \infty$ when $\eta \rightarrow 1^{-}$, this is trivial for $|\theta| \geq(1-\eta)$, so we may assume that $|\theta|<1-\eta$. In this case, we have

$$
-\log \left|\frac{1-\eta t}{1-\eta t e^{i \theta}}\right| \geq \log \left|1+i \frac{\eta t \sin \theta}{1-\eta t}\right|=\frac{1}{2} \log \left(1+\frac{\eta^{2} t^{2} \sin ^{2} \theta}{(1-\eta t)^{2}}\right) \geq \frac{\log 2}{4} \frac{\eta^{2} t^{2} \sin ^{2} \theta}{(1-\eta t)^{2}}
$$

by convexity of the logarithm, and so

$$
\log \left|F_{\eta}(t)\right|-\log \left|F_{\eta}\left(t e^{i \theta}\right)\right| \geq C \alpha(\eta) \frac{\theta^{2}}{(1-\eta)^{2}},
$$

with $C$ an absolute positive constant. Since $\alpha(\eta) \rightarrow \infty$, we may require that

$$
\alpha(\eta) \frac{[\delta(\eta)]^{2}}{(1-\eta)^{2}} \rightarrow \infty
$$

It is clear that the conditions $\delta(\eta) /(1-\eta) \rightarrow 0,(4)$, and (5) are compatible, i.e., that we can find a $\delta$ meeting all three of them. It is also clear that with such a $\delta$, $Q_{\eta, \delta}$ has the required property.

Set

$$
\tilde{v}(z)=\left(\sup \left\{|f(z)|: f \in H_{v}^{\infty},\|f\| \leq 1\right\}\right)^{-1} .
$$

Following [T, we say that a weight $v$ is essential if $v \sim \tilde{v}$, i.e., if there exists a positive constant $C$ such that $v(z) \leq \tilde{v}(z) \leq C v(z)$ for all $z \in \mathbb{D}$. In order to apply the previous lemma to general weights, we need the following lemma. 
Lemma 5. Let $v \in C^{2}(\mathbb{D})$ be a radial weight. If $-\left(1-|z|^{2}\right)^{2} \Delta \log v(z) \rightarrow+\infty$ as $|z| \rightarrow 1^{-}$, then there exists a radial weight $w \in C^{2}(\mathbb{D})$ such that $-\left(1-|z|^{2}\right)^{2} \Delta \log w(z)$ $\nearrow+\infty$ as $|z| \rightarrow 1^{-}$and $u(z)=\frac{v(z)}{w(z)}$ is an essential weight.

Proof. Define

$$
f(z)=-\left(1-|z|^{2}\right)^{2} \Delta \log v(z), \quad z \in \mathbb{D} .
$$

Since $v$ is radial, it follows that $f(z)$ is also a radial function. By assumption, we have $f(t) \rightarrow+\infty$ as $t \rightarrow 1^{-}$. We set $h(z)=\inf _{|z| \leq t<1} f(t)$, and see that $h$ is radial, $h(z) \leq f(z), z \in \mathbb{D}$, and $h(z) \nearrow+\infty$ as $|z| \rightarrow 1^{-}$. We define

$$
F(z)=\frac{h(z)}{\left(1-|z|^{2}\right)^{2}}, \quad z \in \mathbb{D} .
$$

By Proposition 2, there exists a radial weight $w(z)=e^{-\psi(z)}$ such that $\Delta \psi(z)=$ $F(z)$. We have therefore

$$
-\left(1-|z|^{2}\right)^{2} \Delta \log v(z) \leq-\left(1-|z|^{2}\right)^{2} \Delta \log w(z), \quad z \in \mathbb{D},
$$

or, in other words,

$$
-\Delta \log \frac{v(z)}{w(z)} \geq 0 .
$$

The last inequality means that $\frac{w}{v}$ is a log-convex function. We now invoke BDL1. Proposition 7], which implies that $u(z)=\frac{v(z)}{w(z)}$ is then an essential weight.

We are now in a position to prove Theorem 1 . We need only to prove the necessity part, as the sufficiency part is trivial. By Lemma 5, the weight $u(z)=\frac{v(z)}{w(z)}$ is an essential weight, with $w(z)$ being radial and $-\left(1-|z|^{2}\right)^{2} \Delta \log w(z) \nearrow+\infty$ as $|z| \rightarrow 1^{-}$. Since $M_{\varphi}: H_{v}^{\infty} \rightarrow H_{v}^{\infty}$ has closed range, by [BDL2, Lemma 3.1], also $M_{\varphi}: H_{w}^{\infty} \rightarrow H_{w}^{\infty}$ has closed range. Now observe that $u_{1}(z)=\frac{w(z)}{1-|z|^{2}}$ is also an essential weight. Indeed, by the growth property of $\Delta w$, we have $-\Delta \log u_{1}(t) \rightarrow$ $+\infty$ as $t \rightarrow 1^{-}$. Hence there exists $t_{1}>0$ such that

$$
-\Delta \log u_{1}(t)>0 \text { for } t \in\left(t_{1}, 1\right),
$$

and so the same argument as above ensures that $u_{1}$ is essential. Again using BDL2. Lemma 3.1], $M_{\varphi}: H_{w_{1}}^{\infty} \rightarrow H_{w_{1}}^{\infty}$ with $w_{1}(z)=1-|z|^{2}$ has closed range. By [BDL2] Theorem 3.6], $\varphi=h b$, where $h \in H^{\infty}$ is invertible in $H^{\infty}$ and $b$ is a finite union of interpolating Blaschke products. From Lemma 4 it follows that the lower norm of $M_{\varphi_{\eta}}: H_{w}^{\infty} \rightarrow H_{w}^{\infty}$ tends to zero as $|\eta| \rightarrow 1^{-}$. If $b$ were an infinite Blaschke product, then $b$ would have a factor $\varphi_{\eta}$ with $|\eta|$ arbitrarily close to 1 . This leads to a contradiction because $L\left(M_{b}\right) \leq L\left(M_{\varphi_{\eta}}\right)$.

\section{ACKNOWLEDGEMENT}

The first author would like to thank P. Domański for helpful conversations.

\section{REFERENCES}

[A] S. Axler, Multiplication operators on Bergman spaces, J. Reine Angew. Math. 336 (1982), 26-44. MR 84b:30052

[B] K. Bogalska, Multiplication operators on weighted Banach spaces of analytic functions with exponential weights, Bull. Polish Acad. Sci. Math. 49 (2001), 409-416. 
[BDL1] J. Bonet, P. Domański, M. Lindström, Essential norm and weak compactness of composition operators on weighted Banach spaces of analytic functions, Can. Math. Bull. 42 (1999), 139-148. MR 2000d:47052

[BDL2] J. Bonet, P. Domański, M. Lindström, Pointwise multiplication operators on weighted Banach spaces of analytic function, Studia Math. 137 (1999), 177-194. MR 2000m:47042

[BO] B. Berndtsson, J. Ortega-Cerdà, On interpolation and sampling in Hilbert spaces of analytic functions, J. Reine Angew. Math. 464 (1995), 109-120. MR 96g:30070

[L1] D. Luecking, Inequalities on Bergman spaces, Illinois J. Math. 25 (1981), 1-11. MR 82e:30072

[L2] D. Luecking, Multipliers of Bergman spaces into Lebesgue spaces, Proc. Edinburgh Math. Soc. 29 (1986), 125-131. MR 87e:46034

[MS] G. McDonald, C. Sundberg, Toeplitz operators on the disc, Indiana Univ. Math. J. 28 (1979), 595-611. MR 80h:47034

[S] K. Seip, On Korenblum's density condition for the zero sequences of $A^{-\alpha}$, J. Analyse Math. 67 (1995), 307-322. MR 97c:30044

[T] J. Taskinen, Compact composition operators on general weighted spaces, Houston J. Math. 27 (2001), 203-218.

[V] D. Vukotić, Pointwise multiplication operators between Bergman spaces on simply connected domains, Indiana Univ. Math. J. 48 (1999), 793-803. MR 2001b:47052

Faculty of Mathematics and Computer Science, A. Mickiewicz University, ul. MaTEJKI 48/49, 60-769 Poznań, POland

E-mail address: bogalska@amu.edu.pl

Department of Mathematical Sciences, Norwegian University of Science and TechNOLOGY, N-7491 Trondheim, NorWAY

E-mail address: seip@math.ntnu.no 